studies identified a serum trough vancomycin concentration $\geq 15 \mathrm{mg} / \mathrm{L}$ to be an independent risk factor for AKI. To a lesser extent, high dose, duration of treatment and ICU admission were also identified in some studies as risk factors for $\mathrm{v}$ AKI.

Conclusion Incidence of vancomycin-induced kidney injury in children is high. Patients who are high-risk should be identified and monitored more closely in view of reducing the number of cases diagnosed. In particular, concomitant nephrotoxins should be avoided. Well-designed prospective studies are required to determine the mechanism of vancomycininduced kidney injury and the contribution from the above risk factors.

\section{G331(P) ARE WE MISSING PATHOLOGICAL CAUSES OF PROLONGED JAUNDICE BY STREAMLINING OUR INVESTIGATIONS?}

Z Jacob, S Khan. Royal Aberdeen Children's Hospital, NHS Grampian, Aberdeen, UK

\subsection{6/archdischild-2020-rcpch.286}

Aims Prolonged jaundice is common, occurring in $2-15 \%$ of all neonates. NICE guidelines published in 2010 on screening babies with prolonged jaundice were debated and were felt to result in an excess of unnecessary investigations in healthy babies with an otherwise unremarkable examination. These investigations included total and conjugated bilirubin, urine culture, G6PD where ethnically appropriate, full blood count, blood group determination and Coomb's test.

We initially audited 50 of our patient assessments and investigations against advised national guidelines.

Subsequently, we introduced a simple proforma to assist clinicians in assessing for red flag features on history and examination, and reduced recommended investigations to total and conjugated bilirubin only if baby did not have any concerning features on review.

The purpose of this re-audit was to assess whether this change in practice was safe, or had it hampered our ability to identify pathological jaundice, and did it result in better use of resources and improved patient/parent satisfaction.

Methods This prospective re-audit study analysed 105 proformas over a six month period for data on a number of parameters including gestation, age at referral, feeding, weight gain, urine and stool colour.

Outcomes for patients with unconjugated or conjugated hyperbilirubinaemia or those who were admitted were followed up.

The gastroenterology team was consulted for information on any patients who were screened during the audit period and subsequently diagnosed with pathological jaundice.

Results No patients with a diagnosis of pathological jaundice were 'missed' during either audit cycle.

One patient with conjugated hyperbilirubinaemia was diagnosed with alpha-1-antitrypsin deficiency having been flagged up by the streamlined screening process.

Our rate of follow-up investigations increased from 10\% to $24.7 \%$ in the re-audit cycle.

$3.8 \%$ of patients were screened too early. Conclusions The proforma was effective in identifying patients with potentially pathological causes of prolonged jaundice. Reducing investigations resulted in significant savings (potentially $£ 107$ per patient) and better use of NHS resources.

We have since updated our intranet guidelines, aiming to educate practitioners and reduce unnecessary referrals.

We plan to assess whether we can safely reduce our follow-up investigation rate.

\section{G333(P) SERUM BIOMARKERS, BUT NOT DUAL-ENERGY X-RAY ABSORPTIOMETRY (DXA), PREDICT BONE MINERAL DENSITY IN CHILDREN WITH CHRONIC KIDNEY DISEASE}

${ }^{1} \mathrm{AD}$ Lalayiannis, ${ }^{2} \mathrm{NJ}$ Crabtree, ${ }^{3} \mathrm{~V}$ Askiti, ${ }^{3} \mathrm{~A}$ Mitsioni, ${ }^{4} \mathrm{M}$ Fewtrell, ${ }^{5} \mathrm{~A}$ Kaur, ${ }^{6} \mathrm{DV}$ Milford, ${ }^{1} \mathrm{R}$ Shroff. ${ }^{1}$ Paediatric Nephrology, Great Ormond St Hospital and UCL Institute of Child Health, London, UK; ${ }^{2}$ Paediatric Endocrinology, Birmingham Women's and Children's NHS FT, Birmingham, UK; ${ }^{3}$ Paediatric Nephrology, 'P and A Kyriakou' Children's Hospital, Athens, Greece; ${ }^{4}$ Childhood Nutrition Research Centre, UCL Institute of Child Health, London, UK; ${ }^{5}$ Paediatric Nephrology, Manchester University NHS FT, Manchester, UK; ${ }^{6}$ Paediatric Nephrology, Birmingham Women's and Children's NHS FT, Birmingham, UK

\subsection{6/archdischild-2020-rcpch.287}

Introduction Currently available biomarkers and Dual-energy X-ray Absorptiometry (DXA) are thought to be poor predictors of bone mineral density (BMD). The 2017 KDIGO guidelines on CKD-MBD propose using DXA if it will affect patient management. We set out to determine the clinical utility of DXA and routine clinical biomarkers by comparing them with tibial cortical BMD measured by peripheral Quantitative Computed Tomography (pQCT), which clearly defines cortical and trabecular compartments and predicts future fracture risk.

Methods We performed a prospective multi-centre cross-sectional study in 97 children (5-19 years) with CKD stages 4-5 and on dialysis. Children underwent hip and lumbar spine DXA and tibial pQCT. All DXA and pQCT data were expressed as age corrected Z-scores, and lumbar spine DXA was additionally corrected for height (BMAD Z-score). Imaging findings were correlated with routine serum biomarkers.

Results Hip Z-score and lumbar spine BMAD Z-scores correlated significantly with each other $(p<0.001)$. Hip Z-scores and lumbar spine BMAD Z-scores showed a strong correlation with tibial trabecular BMD $(\mathrm{p}<0.001)$, whereas they did not show any correlation with tibial cortical BMD. Neither the hip nor the lumbar spine measures correlated with any biomarkers.

PTH showed a positive correlation with tibial trabecular BMD $(p=0.024)$ and a negative correlation with cortical BMD $(p<0.001)$. Tibial cortical BMD was also associated with alkaline phosphatase $(\mathrm{p}=0.014)$ and total calcium $(\mathrm{p}=0.019)$.

On multivariable linear regression analysis the significant and independent predictors of tibial cortical BMD were PTH $(\beta-0.355, p<0.001)$, alkaline phosphatase $(\beta-0.238$, $\mathrm{p}=0.011)$ and serum calcium $(\beta$ 0.184, $\mathrm{p}=0.052)$, which together predicted $26 \%$ of variability in tibial cortical pQCT.

Conclusions Routinely used biomarkers- calcium, alkaline phosphatase and PTH- when used together are weak to moderate predictors of BMD. No associations were seen with hip or lumbar spine DXA. DXA is not a clinically useful tool and should not be performed routinely. 